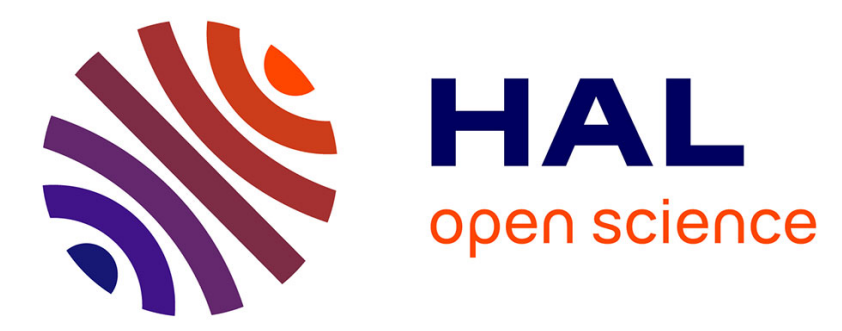

\title{
Assessment of Complement Activation by Nanoparticles: Development of a SPR Based Method and Comparison with Current High Throughput Methods
}

\author{
Jean-Baptiste Coty, Magali Noiray, Christine Vauthier
}

\section{- To cite this version:}

Jean-Baptiste Coty, Magali Noiray, Christine Vauthier. Assessment of Complement Activation by Nanoparticles: Development of a SPR Based Method and Comparison with Current High Throughput Methods. Pharmaceutical Research, 2018, 35 (7), pp.129. 10.1007/s11095-018-2406-5 . hal-03199958

\section{HAL Id: hal-03199958 \\ https://hal.science/hal-03199958}

Submitted on 16 Apr 2021

HAL is a multi-disciplinary open access archive for the deposit and dissemination of scientific research documents, whether they are published or not. The documents may come from teaching and research institutions in France or abroad, or from public or private research centers.
L'archive ouverte pluridisciplinaire HAL, est destinée au dépôt et à la diffusion de documents scientifiques de niveau recherche, publiés ou non, émanant des établissements d'enseignement et de recherche français ou étrangers, des laboratoires publics ou privés. 


\title{
Assessment of complement activation by nanoparticles: development of a SPR based method and comparison with current high throughput methods
}

\author{
Jean-Baptiste Coty, Magali Noiray, Christine Vauthier*
}

Institut Galien Paris-Sud, CNRS, Univ. Paris-Sud, Université Paris-Saclay, 5 rue Jean-Baptiste Clément, 92290 Châtenay-Malabry, France

Running Head: SPR for complement activation by nanoparticles

Published in: Pharm Res 2018;35:129. https://doi.org/10.1007/s11095-018-2406-5

*Correspondence: Dr. Christine Vauthier, CNRS UMR 8612, Institut Galien Paris Sud, Univ. Paris-Sud, Université Paris-Saclay, 5, rue Jean-Baptiste Clément, 92296 Châtenay-Malabry, France. Fax: +33 146835946. E-mail: christine.vauthier@u-psud.fr

\begin{abstract}
Purpose: A Surface Plasmon Resonance chip (SPR) was developed to study the activation of complement system triggered by nanomaterials in contact with human serum, which is an important concern today to warrant safety of nanomedicines. Methods: The developed chip was tested for its specificity in complex medium and its longevity of use. It was then employed to assess the release of complement fragments upon incubation of nanoparticles in serum. A comparison was made with other current methods assessing complement activation ( $\mu$ C-IE, ELISA). Results: The SPR chip was found to give a consistent response for $\mathrm{C} 3 \mathrm{a}$ release upon activation by nanoparticles. Results were similar to those obtained by $\mu \mathrm{C}$-IE. However, ELISA detection of iC3b fragments showed an explained high nonspecific background. The impact of sample preparation preceding the analysis was assessed with the newly develop SPR method. The removal of nanoparticles before analysis showed an important modification in the obtained response, possibly leading to false negative results. Conclusion: The SPR chip developed in this work allows for an automated assessment of complement activation triggered by nanoparticles with possibility of multiplexed analysis. The design of the chip proved to give consistent results of complement activation by nanoparticles.
\end{abstract}

Key-words: Complement system - Nanoparticles - Surface plasmon resonance - Automated method 


\section{Abbreviations}

ASTM: American Society for Testing and Materials

CARPA: Complement Activation Related Pseudo Allergy

CVF: Cobra venom factor

ELISA: Enzyme-linked immunosorbent assay

HAGG: Heat-activated gamma globulin

IBCA: Isobutylcyanoacrylate

ISO: International Organization for Standardization

NSB: non-specific binding

NM: Nanomedicine

NP: Nanoparticle

RSD: Relative standard deviation

RU: Resonance unit

SPR: Surface plasmon resonance

2D-IE: Two-dimensional immunoelectrophoresis

$\mu \mathrm{C}$-IE: Multi-crossed 2D immunoelectrophoresis 


\section{Introduction}

The complement system is a major effector of the human innate and adaptive immunity. Consisting of 35 soluble blood proteins, this system can be activated through proteolytic cleavages that are arranged in a cascade when a foreign body is detected (bacteria, virus, nanomaterial). The triggering of this cascade leads to inflammation reactions by the release of anaphylatoxins (C3a, C5a) as well as enhancement of phagocytic activity and formation of lysis complex $[1,2]$. In the case of nanomedicines (NMs) used upon intravenous administration, complement proteins are the first barrier of immunity encountered by the nanomaterial in human blood. The biodistribution, circulation time, or the possible induced toxicity of these nanomaterials can be influenced by their interaction with the complement system $[3,4]$. Indeed, the interaction of NMs with complement proteins triggers this cascade that can lead to severe adverse reactions referenced in the CARPA (Complement Activation Related Pseudo Allergy) [5-7] and has even been linked to an enhancement of cancer tumor growth [8,9].

Despite these facts, complement activation has not been positioned as a priority assessment in NMs design and in their clinical batch release. While assays are recommended for complement activation of material devices by ISO (International Organization for Standardization) [10] and ASTM (American Society for Testing and Materials) [11], only few recommendations for the complement activation evaluation of intravenous drug are existing $[12,13]$. The development of a direct and automatable method on this purpose could favor its assessment hence improving the safety and knowledge about NMs links with the complement system. Today, the reference method used for in vitro complement activation assessment is ELISA (Enzyme-linked immunosorbent assay) [6,14-17]. This method was standardized and marketed available kits allow the detection of different specific fragments of the complement cascade (iC3b, Bb, C4d, SC5b9). However, this method suffers from its high cost. A method such as two-dimensional immunoelectrophoresis (2D-IE) allows a cheaper assessment of complement activation assessment with a global vision of $C 3$ and its fragments $[15,18]$. This method was recently improved to be applicable on a higher throughput (multi-crossed 2D immunoelectrophoresis: $\mu \mathrm{C}-\mathrm{IE})$, but still remains time consuming. The use of a fully automatable technique such as surface plasmon resonance (SPR) to detect complement fragments in serum is another way to deal with complement assessment. SPR is widely used for affinity and kinetics studies between a ligand and a given substrate. NM-protein interaction has already been studied by SPR by grafting the NMs onto the gold chip before flowing the biological medium and looking for interactions and kinetics of association-dissociation [19-21].

However, a major hurdle when working with complement system is the matrix complexity such as encountered in human serum where the non-specific protein binding is a real problem faced in SPR. Development of strategies to overcome this major drawback are emerging but require the engineering of complex chips [22-24]. This typically prevents many applications from being studied by SPR. Nevertheless, some groups already worked on complement detection by SPR with different approaches where the tested biomaterial is generally coated on the chip [25-27], that limits the possibilities of high throughput analysis of several distinct biomaterials. 
Added to these aspects, antibody regeneration is another critical issue when working with SPR. In previously mentioned studies, specific antibodies were flowed on the surface and their binding on complement proteins interacting with the biomaterial surface increased the signal. This procedure supposes a high consumption of antibodies, hence a high cost. The other way is to coat specific antibodies on the chip and then flow activated serum for binding of specific fragments. The difficulty of this procedure is to regenerate coated antibodies to keep their functionality for other samples [2830]. This was a challenge taken into account in our method development.

Finally, the way to detect complement activation by looking for a specific fragment is prone to possible artefacts such as adsorption of this specific component on the nanoparticle (NP) thus preventing its detection. A fragment of protein C3 that is quickly released is the C3a fragment (9000 Da), which acts as an anaphylatoxin, triggering inflammatory reactions. Several works reported a possible adsorption of this fragment on polymer surfaces, thus biasing their quantification when assessed in the serum supernatant [31]. The phenomenon was first evidenced on polymers forming dialysis membrane such as polyacrylonitrile or cellulose acetate [32,33]. Then, their adsorption onto different types of grafted polymers were studied by Montdargent et al. [34]. They showed that adsorption on polymer surface was occurring with anaphylatoxins $\mathrm{C} 3 \mathrm{a}$ and $\mathrm{C} 5 \mathrm{a}$ for neutral, anionic and cationic polymer surfaces. The adsorption greatly depended on the chemical nature of the polymer substituent group. In the present work, we tested different NPs with the same chemical composition but different surface conformations and showed that this parameter was of importance too regarding C3a adsorption. These results were further put in relation with possible bias occurring with current established protocols for complement activation assessment.

The developed SPR method was confronted to standardized methods already used for complement activation assessment. Thus, the approach of this paper allowed a comparison between three methods (ELISA, SPR and $\mu \mathrm{C}-\mathrm{IE}$ ). Such a comparison has never been reported in the literature. Indeed, complement activation is generally evaluated only with commercially available assays, mostly ELISA.

The purpose of this work was to develop a SPR-based method for a simplified and cost-effective assessment of complement activation by NMs. Results obtained with this method were challenged with standardized and robust methods, namely ELISA and $\mu$ C-IE. Finally, a complementary study aimed to evaluate the possible bias introduced by the presence of NPs in the medium and thus adapt the optimal conditions for reliable assays. To support this study, a series of five model NPs of the same chemical composition but bearing distinct surface density and conformation chains were used.

\section{Material and methods}

\section{II.1. Materials}

Chemicals: Isobutylcyanoacrylate (IBCA) was provided by Orapi (Saint-Vulbas, France). Tricine, Tris base (Sigma 7-9 ${ }^{\circledR}$ ), sodium chloride, EDTA sodium salt, dextran $66.7 \mathrm{kDa}$, and bromophenol blue were purchased from Sigma (Saint-Quentin-Fallavier, France). Dextran 17.7, cerium (IV) ammonium nitrate, and hydrochloric acid were supplied by Fluka (Saint-Quentin-Fallavier, France). Nitric acid was provided 
by Prolabo (Paris, France). Calcium lactate, glacial acetic acid, coomassie brilliant blue R-250 were supplied by Thermo Fisher Scientific (Villebon-sur-Yvette, France). Gel-Fix ${ }^{\mathrm{TM}}$ for agarose ( $265 \times 150 \mathrm{~mm}$ ) was obtained from Serva Electrophoresis (Heidelberg, Germany). Amine coupling kit, Series S Sensor Chip CM5, HBS-EP+ buffer 10X, NSB reducer and HCl-Glycine pH 2.5 were obtained from GE Healthcare (Velizy-Villacoublay, France). All chemicals were of reagent grade and used as purchased.

Biologicals: Goat IgG (purity > 80\%) was obtained from Sigma (Saint-Quentin-Fallavier, France). Polyclonal anti-human C3 antibody raised in goat was purchased from Fitzgerald antibodies (Acton, USA). Monoclonal anti-human C3a neoepitope produced in mouse came from antibodies-online $\mathrm{GmbH}$ (Aachen, Germany). Serum was prepared from human plasma obtained from healthy donors from Etablissement Français du Sang (EFS, agreement \# 14/EFS/041) (Rungis, France). Heat-activated gamma globulin (HAGG) and Cobra Venom Factor (CVF) were purchased from TECO Medical (Rambouillet, France) to be used as positive controls for the activation of the complement system. Proteins C3b (purity $>90 \%$ ) and C3c (purity $>80 \%$ ) were provided by Acris Antibodies $\mathrm{GmbH}$ (Herford, Germany). Human complement C3a (purity > 98\%) was purchased from Merck Calbiochem ${ }^{\circledR}$ (Fontenay-sous-Bois, France). Human complement C3 protein (purity $>85 \%$ ) and Microvue ${ }^{\mathrm{TM}}$ complement iC3b EIA kit were purchased from Teco Medical (Rambouillet, France).

\section{II.2. Methods}

\section{II.2.1.NPs synthesis and characterization}

Five types of nanoparticles were prepared from well described emulsion polymerization methods of IBCA with dextran. The method and characteristics of the nanoparticles were thoroughly described in our previous works [35-37]. In this work, three NPs were obtained from radical redox emulsion polymerization ( $R 1, \mathrm{R} 2$ and $\mathrm{R} 3$ ), two others were prepared by anionic emulsion polymerization ( $\mathrm{A} 1$ and A2). They differ by the density and conformation of dextran chains grafted on their surface as illustrated later in this article. They display different capacities to activate the complement system [37]. The table 1 depicts the different characteristics of nanoparticles used in this work and the figure 1 shows transmission electron micrographs of the nanoparticles. The characteristics of the nanoparticles were consistent with those presented in previous work [35-37].

The hydrodynamic diameter of nanoparticles was measured at $20^{\circ} \mathrm{C}$ by dynamic light scattering (DLS) using a ZetaSizer Nano ZS90 (Malvern Instruments, Orsay, France). Prior analysis, dispersions were diluted in MilliQ water at $1 / 200$ (NPs R1 and R2), 1/100 (NPs A1), 1/50 (NPs R3) and 1/20 (NPs A2). Size distributions are shown on figure $\mathrm{S} 1$ in the supplemental information. Surface charge was evaluated through the determination of an apparent zeta potential (zeta potential) deduced from measurements of the electrophoretic mobility of the nanoparticles by electrophoresis light scattering (ELS) using the ZetaSizer Nano ZS90 (Malvern Instruments, Orsay, France). The Smoluchowski's model was used to calculate the zeta potential without applying a correction for the surface conductivity. Nanoparticle dispersions were diluted in $1 \mathrm{mM} \mathrm{NaCl}$ solution to perform this analysis. Transmission electron micrographs were obtained from observations of grids with a Jeol JEM 1400 electron microscope operating at $120 \mathrm{KV}$ and equipped with a camera ORIUS SC1000A2 (Electron Microcopy 
Facility of I2BC, Gif sur Yvette, France). A $2 \mu \mathrm{L}$ sample of nanoparticle dispersion diluted in filtered MilliQ ${ }^{\circ}$ water was deposited on a formvar-carbon cupper grid for electron microscopy and let to dry in a dust free environment. No staining was applied.

Table 1: Physicochemical characteristics of model nanoparticles used for complement activation. Hydrodynamic diameter, zeta potential, concentration, and schematic representation of the NPs.

\begin{tabular}{|c|c|c|c|c|c|}
\hline NPs & $\begin{array}{l}\text { Hydrodynamic } \\
\text { diameter }{ }^{*} \\
(\mathrm{~nm})\end{array}$ & $\begin{array}{l}\text { Polydispersity } \\
\text { index* }\end{array}$ & $\begin{array}{c}\text { Zeta } \\
\text { potential } \\
* *(\mathrm{mV})\end{array}$ & $\begin{array}{l}\text { Concentration } \\
* * *(\mathrm{mg} / \mathrm{mL})\end{array}$ & $\begin{array}{c}\text { Schematic } \\
\text { representation } \\
\text { [37] }\end{array}$ \\
\hline R1 & $286 \pm 14$ & 0.030 & $-4 \pm 2$ & $38 \pm 2$ & \\
\hline R2 & $208 \pm 4$ & 0.041 & $-10 \pm 1$ & $44 \pm 2$ & \\
\hline R3 & $281 \pm 6$ & 0.061 & $-5 \pm 2$ & $47 \pm 4$ & \\
\hline A1 & $88 \pm 2$ & 0.105 & $-5 \pm 1$ & $37 \pm 2$ & \\
\hline A2 & $159 \pm 10$ & 0.040 & $-4 \pm 2$ & $13 \pm 3$ & \\
\hline \multicolumn{6}{|c|}{$\begin{array}{l}\text { *by dynamic light scattering (DLS); }{ }^{* *} \text { by electrophoretic } \\
\text { light scattering (ELS); }{ }^{* *} \text { by gravimetry. }\end{array}$} \\
\hline
\end{tabular}


Figure 1: Transmission electron micrographs of the 5 dispersions of nanoparticles. Samples were deposited on carbon-formvar coated grids and let to dry in a dust free environment. Observations were performed with a Jeol JEM 1400 electron microscope operating at $120 \mathrm{KV}$ and equipped with a camera ORIUS SC1000A2 (Electron microcopy facility of I2BC). No staining was applied during preparation of the grids.

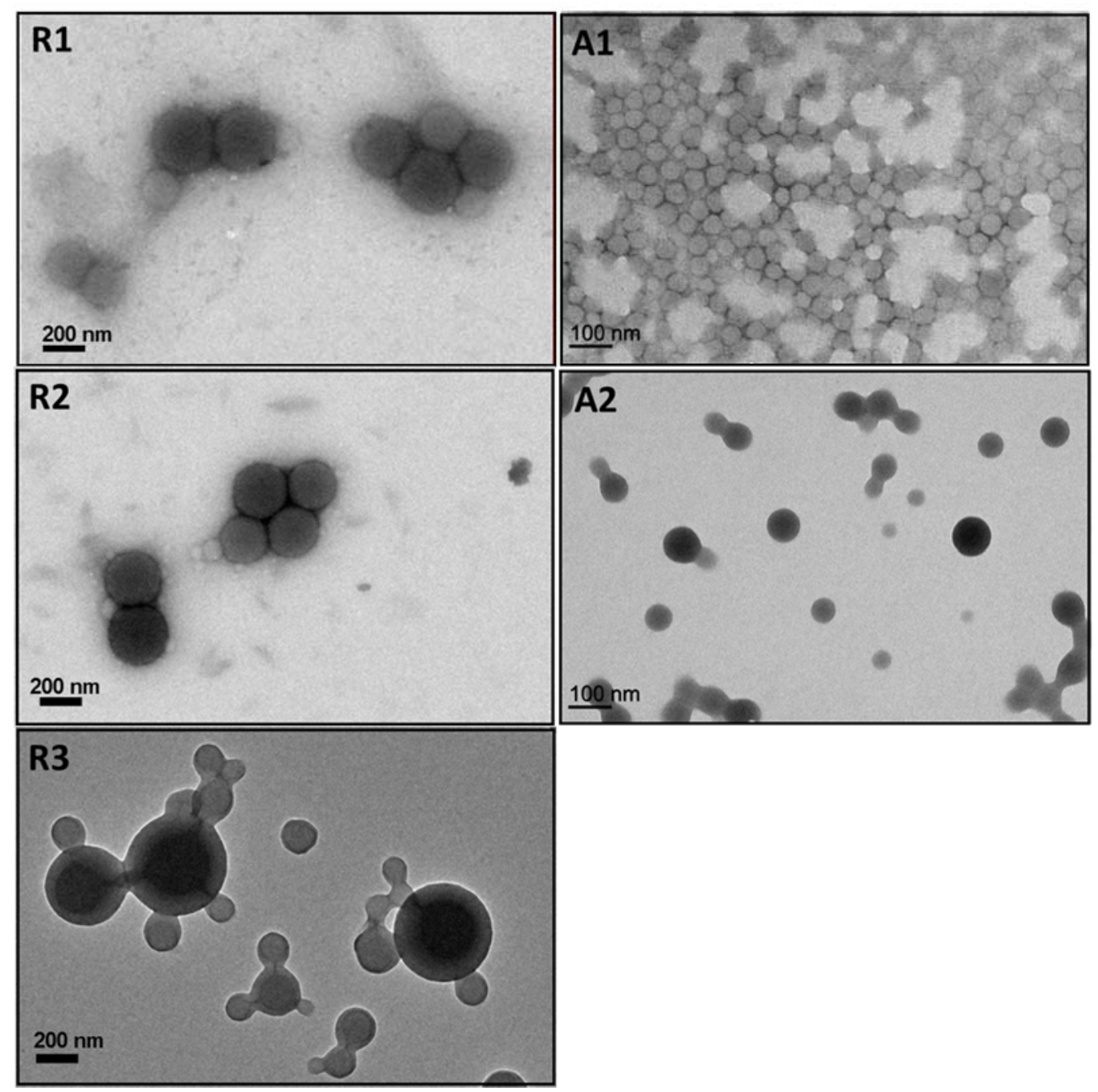

Sample preparation for complement activation assessments

The five types of NPs were assessed at two different concentrations (1000 and $3000 \mathrm{~cm} / \mathrm{mL}$ ) for complement activation. The incubation was prepared as described previously [20] by diluting human serum (1:4) with VBS ${ }^{2+}$ buffer (1:4) and NPs aqueous dispersion at the desired concentration (1:2) or water for controls. The final incubation volume was $100 \mu \mathrm{L}$. For the negative control, $\mathrm{VBS}^{2+}$ was replaced by VBS EDTA, that inhibits complement activation. The positive control was obtained by introducing CVF $(5 \mu \mathrm{L})$, a strong activator of the complement cascade, in the incubation medium filled 
with water $(45 \mu \mathrm{L})$ to reach the final volume of $100 \mu \mathrm{L}$. Medium activation was obtained by introducing HAGG $(5 \mu \mathrm{L})$ in the incubation medium as described above for CVF control.

The incubation mixture was left at $37^{\circ} \mathrm{C}$ for an hour. After incubation, samples were kept in ice few minutes until analysis. Each sample was analyzed in triplicate by SPR and $\mu \mathrm{C}$-IE and in duplicate by ELISA.

\section{II.2.2. Surface Plasmon Resonance (SPR)}

\section{II.2.2.1. Antibody immobilization}

SPR measurements were performed on a Biacore T100 instrument with a Series S sensor chip CM5 equipped with 4 channels (GE healthcare Life Sciences, Vélizy, France). Antibodies were immobilized onto the chip via a standard amine coupling consisting in a successive injection of 1-ethyl-3-(3dimethylaminopropyl)carbodiimide hydrochloride (EDC) (75 mg/mL, $420 \mathrm{sec}, 5 \mu \mathrm{L} / \mathrm{min}$ ), Nhydroxysuccinimide (NHS) $(11.5 \mathrm{mg} / \mathrm{mL}, 420 \mathrm{sec}, 5 \mu \mathrm{L} / \mathrm{min})$ and antibody $(20 \mu \mathrm{g} / \mathrm{mL})$ in $10 \mathrm{mM}$ acetate buffer pH 4.5 (420 sec, $10 \mu \mathrm{L} / \mathrm{min}$ ) to reach an immobilization level of 10,000 RU (Resonance Unit). The remaining blank were filled with ethanolamine- $\mathrm{HCl} \mathrm{pH} 8.5(1 \mathrm{M})$ to prevent for residual non-specific binding (NSB). NSB was assessed on a channel bearing a non-relevant antibody and another channel with a blank surface.

\section{II.2.2.2. Specificity assays and control serums}

Pure C3 (185 kDa), C3b (176 kDa) and C3c (140 kDa) proteins were tested at $140 \mu \mathrm{g} / \mathrm{mL}$ in HBS-EP+ buffer. Pure C3a $(9,1 \mathrm{kDa})$ was analyzed at $70 \mu \mathrm{g} / \mathrm{mL}$ in HBS-EP+ buffer. Control serum samples incubated as described above were then diluted by 12.5 in the buffer (HBS-EP+/NSB reducer 9:1) to reach a final dilution factor of 50 for human serum $(4 \times 12.5)$. All samples were analyzed sequentially on the different channels at $25^{\circ} \mathrm{C}$ in the following conditions: flow rate: $40 \mu \mathrm{L} / \mathrm{min}$; contact time: 60 $\mathrm{sec}$; dissociation time: $120 \mathrm{sec}$. Channels were then regenerated with a two steps procedure: (i) Glycine/ $\mathrm{HCl} \mathrm{pH} \mathrm{2.5:} \mathrm{contact} \mathrm{time:} 30 \mathrm{sec}$ at $40 \mu \mathrm{L} / \mathrm{min}$, (ii) $\mathrm{NaOH} 1 \mathrm{mM}$ (30 sec at $40 \mu \mathrm{L} / \mathrm{min}$ ). The SPR signal obtained is proportional to the mass of analyte interacting with the surface. The quantification of analyte binding on the channel was expressed in $\mathrm{RU}\left(1 \mathrm{RU}=1 \mathrm{pg} / \mathrm{mm}^{2}\right)$ and the report point was taken 10 seconds after the beginning of the dissociation. An example of SPR sensorgram is available in supplementary information S2.

\subsubsection{NP-serum studies}

All serums were prepared and analyzed as described in the previous paragraph. Analyzes were performed on the samples directly after incubation without additional step to remove the nanoparticles. In few experiments, nanoparticles were removed from the samples prior to analysis to study the bias possibilities caused by the removal of the NPs. In this part, the incubated serums were centrifuged at $4^{\circ} \mathrm{C}$ for $45 \mathrm{~min}$ at $16.1 \mathrm{rcf}$ (relative centrifugal force) with an Eppendorf centrifuge 5415R 
(Eppendorf, Montesson, France) prior to the dilution. Then, the supernatant was recovered for dilution preparation and analysis. Analyzed sera corresponding to these samples were identified as "samples without nanoparticles" in the results and discussion section.

\section{II.2.3.Multi-crossed 2D Immuno-electrophoresis ( $\mu \mathrm{C}$-IE)}

Multi-crossed 2D immune-electrophoresis was performed as described previously [20,38]. In summary, a $1 \%$ agarose gel plate was prepared on Gel-Fix ${ }^{\mathrm{TM}}$ alternating plain agarose gel bands (0.8 $\mathrm{cm}$ wide) and agarose gel bands containing polyclonal anti-C3 antibody ( $2.2 \mathrm{~cm}$ wide) as explained in a previous work (20). Incubated samples were deposited on wells formed in the agarose gel bands to run the first dimension allowing the separation of proteins according to their molecular weight (600 $\mathrm{V}, 16 \mathrm{~mA}$ ). Then, the plate was turned by $90^{\circ}$ to allow the second-dimension electrophoresis leading to the immunoprecipitation of $\mathrm{C} 3$ protein and its fragments in the gel bands containing the polyclonal anti-C3 antibody (500 V, $12 \mathrm{~mA})$. Gel was then dried and stained with Coomassie blue prior to analysis of $\% \mathrm{C} 3$ activation.

\section{II.2.4.ELISA}

Elisa was performed following the instructor procedure. Briefly, the sample was diluted by 100 in the specimen diluent provided by the supplier. Then, the sample was deposited and incubated at room temperature for $30 \mathrm{~min}$. The plate was thoroughly washed with the wash solution. The iC3b reagent was then added and left for 30 min before being washed. Substrate was added for 30 min before adding the stop solution. The ELISA plate was read at $405 \mathrm{~nm}$ using LT-5000MS Elisa plate reader (Labtech France, Palaiseau).

\section{Results}

\section{III.1. Setup of a regenerable SPR chip for the direct assessment of complement activation by nanoparticles}

\section{III.1.1. Antibody immobilization}

The SPR chip was grafted with a goat IgG with no affinity for complement fragments on channel 1 to give a non-specific reference channel. Antibody directed against human C3a neo-epitope was coated on channel 2 at around 10,000 RU. Anti-human C3 polyclonal antibody was coated on the third channel at the same level of immobilization. Channel 4 exposed the raw surface of the chip for another reference with no affinity. In this chip, channels 1 and 4 could highlight possible non-specific adsorption while channels 2 and 3 could evaluate specific fragments of the complement system. It was assumed that the non-specific adsorption behavior monitored on channel 1 grafted with the non-specific goat IgG was consistent to that of channels 2 and 3, grafted with antibodies specific to complement fragments. 


\section{III.1.2. Specificity assays and control serums}

At first, the specificity of the different channels towards different protein C3 fragments was assessed to ensure their relevance for the intended use. Results are depicted in figure $2 \mathrm{~A}$. The channel 1 showed no affinity for any of the tested proteins. Channel 2 was specific to C3a released fragment, confirming the ability of the antibody to specifically detect the neo-epitope of C3a. Channel 3 could detect both C3 protein, C3b and C3c fragments, but not C3a.

Figure 2: Evaluation of the SPR chip performance. A: Evaluation of antibodies specificities on SPR chip channels. B: channel response to control sera with different level of complement activation. C: Study of the longevity of specific antibodies to C3 fragments grafted on channels 2 and 3 . Channel 1: Goat IgG; Channel 2: Anti-C3a monoclonal antibody; Channel 3: Anti-C3 polyclonal antibody.
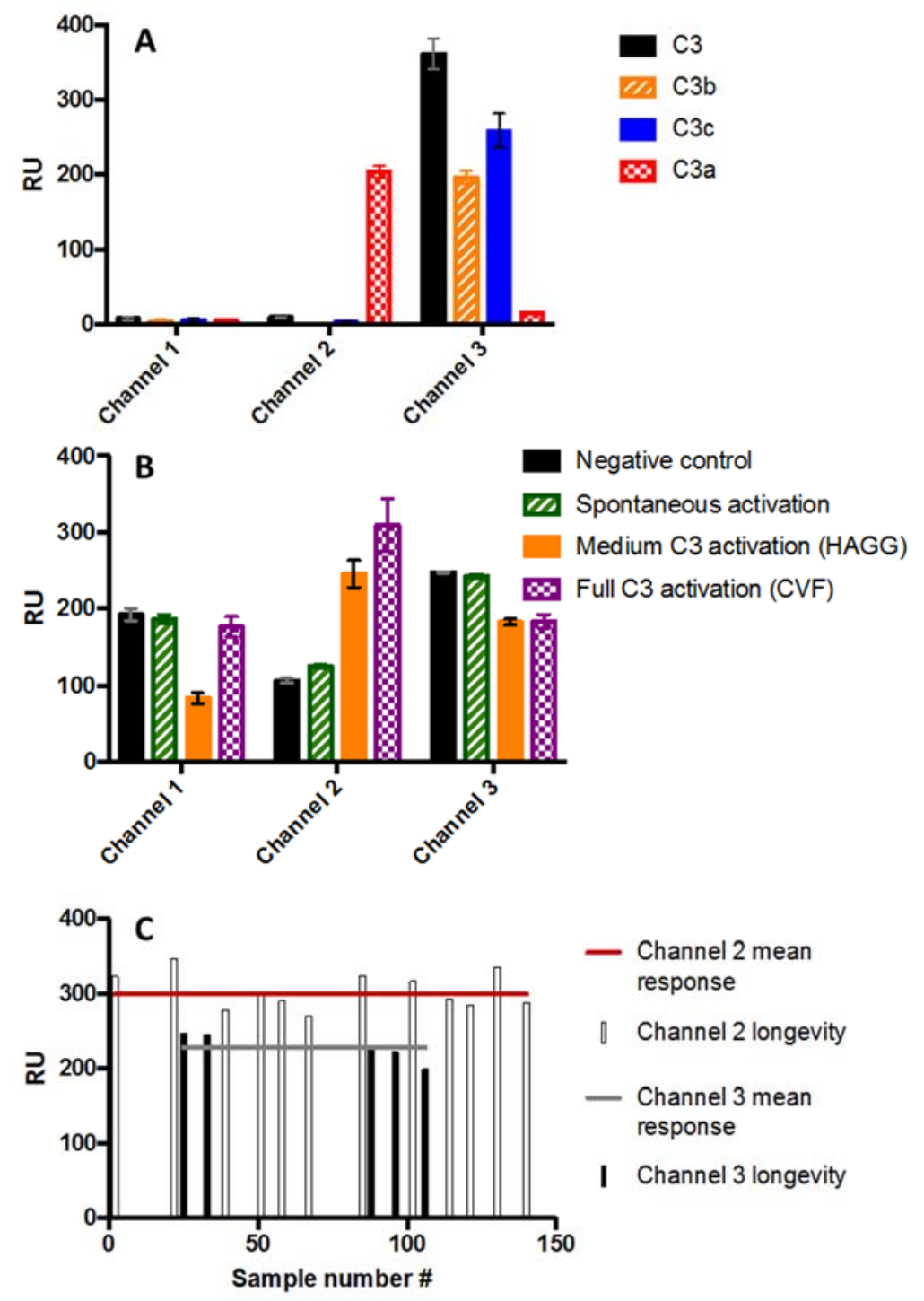
Then, these channels were tested for their ability to detect these specific fragments in sera with different levels of C3 activation obtained from control samples made with known activators (HAGG, CVF) and inhibitors (EDTA) of complement activation. Flow conditions, buffer and sample dilution described in the previous part were optimized in order to give a relevant signal on the response for all channels. Results are presented in figure $2 \mathrm{~B}$. CVF is a positive control that produces complement $\mathrm{C} 3$ full fragmentation releasing $\mathrm{C} 3 \mathrm{a}$ and $\mathrm{C} 3 \mathrm{~b}$ fragments. The results indicated that non-specific binding was quite important with serum, showing an important background level on channel 1. Nevertheless, channel 2 showed an ability to distinguish activated C3 samples from negative controls. Non-specific binding found on negative control was around $100 \mathrm{RU}$ while the fully activated serum gave a response above $300 \mathrm{RU}$. In contrast with our previous hypothesis, we have found that the level of non-specific signal depended on each channel according to the grafted antibody. The variation of non-specific binding between the different channels was nevertheless similar. On channel 3, we observed a decrease of the signal for $\mathrm{C} 3$ activated samples meaning a decrease of the mass of protein binding to the immobilized antibody. The decrease is substantial, but the amplitude is less important than for channel 2 between negative and positive full activated controls.

\section{III.1.3. Chip longevity}

The regeneration was optimized to permit a sustained use of coated antibodies. The longevity of channel 2 was assessed with the fully activated sample (CVF) containing a maximum amount of C3a. The channel 3 longevity was monitored with the negative control (VBS EDTA) containing maximum of C3 native protein (Figure 2C). A quite constant value for C3a detection in a fully activated control serum was obtained on Channel 2 along the analysis of almost 150 samples. A slight variation was observed between the different runs, but the relative standard deviation (RSD) for the positive control on channel 2 over the 150 analyses was $<10 \%$. The behavior of channel 3 was similar to channel 2 showing good performance after regeneration. The signal tended to decrease slightly after nearly 100 analyses. A similar RSD value below $10 \%$ was also obtained on channel 3 for the longevity study.

\section{III.2. SPR, $\mu \mathrm{C}$-IE and ELISA comparison of the assessment of complement activation produced by NPs}

The activation profile of five poly-IBCA NPs already well described were compared using three methods for complement activation assessment: SPR developed in this paper, $\mu \mathrm{C}-\mathrm{IE}[20]$ and ELISA. The results of activation at two given surface concentrations of NPs $\left(1000\right.$ and $\left.3000 \mathrm{~cm}^{2} / \mathrm{mL}\right)$ are presented in figure 3.

The activation of the complement cascade was respectively assessed by the detection of C3a fragment by SPR (channel 2), iC3b fragment by ELISA and the balance between native $\mathrm{C} 3$ and released fragment (C3b, C3c) by $\mu \mathrm{C}$-IE. All these determinations were linked to the cleavage of the C3 protein of the complement system. 
Figure 3: Complement activation by five types of NPs assessed by $\mu C-I E, S P R$ (channel 2) and ELISA. Serum was exposed to $1000 \mathrm{~cm} 2 / \mathrm{mL}(A)$ and $3000 \mathrm{~cm} 2 / \mathrm{mL}$ (B) of nanoparticles.

Indicates the activation of $C 3$ protein ( $(B)$, leading to the release of protein fragments $C 3 b$ ( ) and $\mathrm{C} 3 \mathrm{a}(\mathrm{m})$.
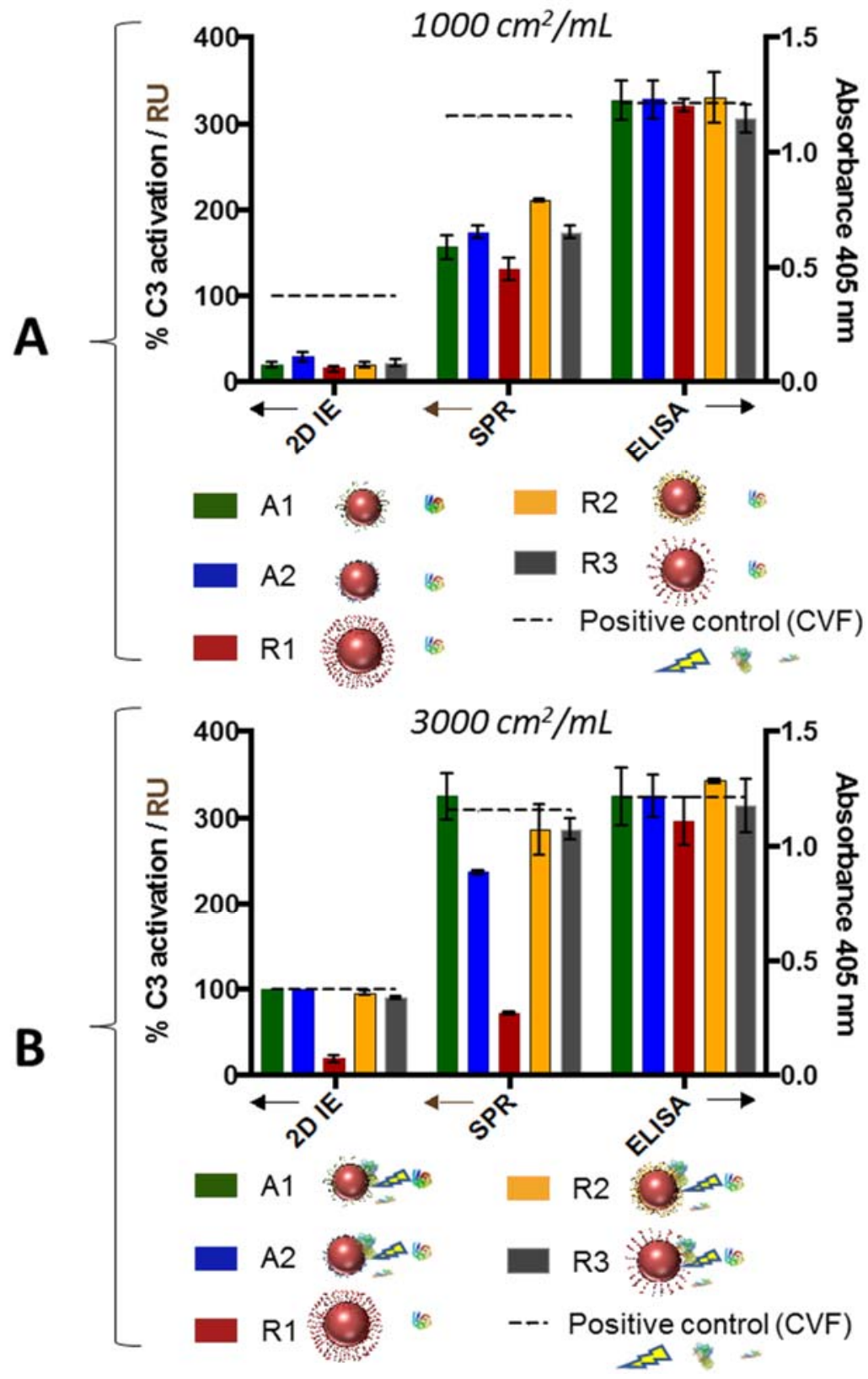

$\mu \mathrm{C}$-IE was used as our reference method, giving reproducible results of complement activation based on the monitoring of the cleavage of the $\mathrm{C} 3$ protein as demonstrated in previous works $[20,38]$. At $1000 \mathrm{~cm}^{2} / \mathrm{mL}$, the level of activation expressed in $\mu \mathrm{C}$-IE was quite low (below $30 \%$ ) for all tested NPs. The same level was obtained for C3a fragment detected by SPR when comparing with values of negative and positive controls shown in figure 2 . At $3000 \mathrm{~cm}^{2} / \mathrm{mL}$ where $A 1, A 2, R 2$ and R3 NPs are known to strongly activate the complement system [38], SPR measurements of C3a fragments released 
gave consistent results with $\mathrm{C} 3$ cleavage observed by $\mu \mathrm{C}$-IE. The activation reached the same level as fully activated CVF control for NPs A1, A2, R2, R3 while R1 was non-activating. The ELISA detection of iC3b fragment gave high values of absorbance in all cases. Tendencies that might emerge from this method were counterbalanced by standard deviations that prevent from a possible distinction in terms of iC3b released.

\section{III.3. Investigation of possible bias involved in complement activation evaluation methods}

A study about sample preparation's influence was done with the SPR technique to evaluate possible bias involved in complement activation evaluation. To perform this investigation, nanoparticles were removed from the incubation medium by centrifugation prior to SPR analysis and the results were compared with those obtained analyzing the incubation medium still containing the NPs. The SPR allows the sample to be flowed onto the channels for protein specific recognition. Released proteins are thus captured. In this part, we compared the effect of removal of NPs prior to SPR analysis. Results were depicted in figure $4 \mathrm{~A}$.

Figure 4: Comparison of C3a level detection by SPR before and after removal of NPs by centrifugation. $A$ : on samples incubated with NPs for one hour. B: On samples fully activated by CVF and then incubated with NPs for one hour.

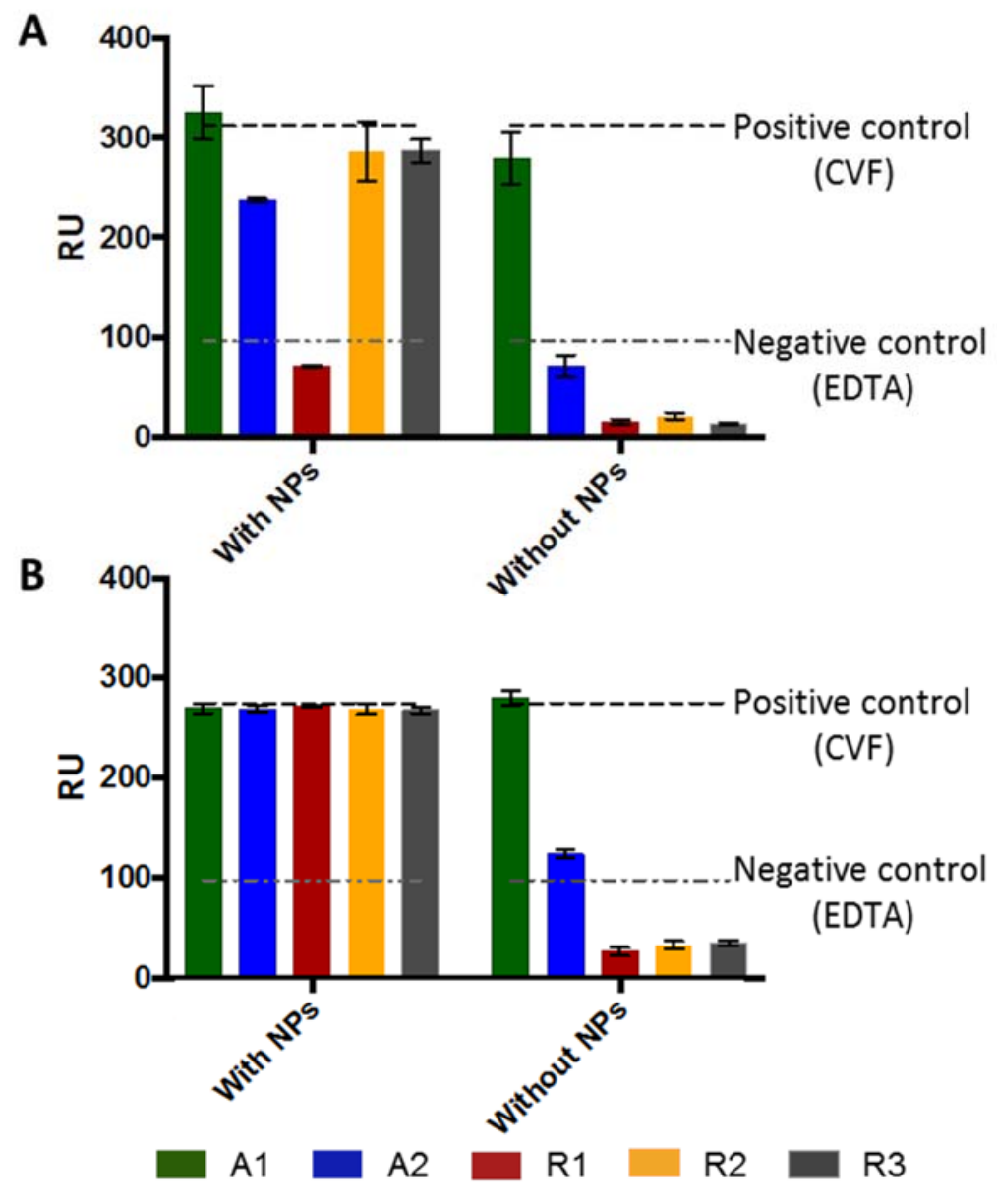


An important decrease of C3a signal was observed after removal of most NPs excepted for A1, which seemed to not disturb the determination of the C3a level by SPR (Figure 4A). The observed effect was particularly pronounced for A2, R2 and R3 NPs where C3a signal dramatically felt after removal of NPs. NP R1, while producing only a little amount of C3a fragment also seemed to impact on its detection when they were removed prior to analysis.

Another assay was carried out with serum that was first fully activated with CVF, leading to a full conversion into C3a, and then incubated for one hour with nanoparticles (Figure 4B). The purpose of this experiment was to investigate whether results from analysis of this fully activated serum could be disturbed by incubation and removal of nanoparticles due to possible adsorption of the C3a protein. The signal on Channel 2 for C3a response was monitored before and after removal of the NPs (Figure $4 B)$. Observations made from the first study depicted in figure $4 \mathrm{~A}$ were confirmed with this experiment. A strong decrease of C3a level was observed upon removal of NPs for A2, R1, R2, and R3, whereas A1 removal from the incubation medium did not significantly influence the level of detected C3a. A strong adsorption of the C3a fragment onto NPs was thus evidenced for A2, R1, R2 and R3. It is worth noting that $\mathrm{A} 2$ does not reduce the signal at the same level as R1, R2 and R3 NPs. Conversely, the response on channel 1 , reference for non-specific binding, and channel 3, bearing anti-C3 polyclonal antibody, were almost unchanged between these two types of samples (see Supplementary Information S3). This meant that a strong adsorption of random serum proteins onto NPs was not at the root of the signal decrease observed on channel 2 , but rather a very specific adsorption of C3a onto NPs.

\section{Discussion}

A SPR chip bearing C3 specific antibodies for the assessment of complement activation by nanoparticles was optimized in this work. The detection of complement activation focused on C3 protein that is at the heart of the complement cascade. Monitoring the activation of this protein allows the assessment of global complement activation. In the developed chip, an antibody against C3a neoepitope and a polyclonal anti-C3 antibody were used. The first allowed a specific detection of a released fragment resulting from $\mathrm{C} 3$ activation. The second permitted assessment of native C3 variation. We could observe a decrease of the signal on channel 3 grafted with polyclonal anti-C3 antibody upon complement activation (Figure 2B), meaning a decrease of the mass of protein captured on the surface. This was consistent with the fragmentation of the native C3 (185 kDa) into smaller fragments recognized by this channel (C3b: $176 \mathrm{kDa}$ and C3c: $139 \mathrm{kDa}$ ). Then, the C3a fragment was used as a finest revelator of $\mathrm{C} 3$ cleavage and thus complement cascade activation.

The aim was to develop an automatable method for a high throughput analysis of samples. The requirements for such an application were firstly to check the antibody specificity with pure proteins before testing their response to different level of activated serums. Specificity of antibodies grafted on channels 2 and 3 could be established using purified $C 3$ proteins and various corresponding fragments produced during activation. Another requirement was the wish to limit costs associated with the analysis. Usually, SPR analysis require the grafting of an antibody on the chip for each sample measurement. The regeneration steps destroy all proteins and then the primary antibody grafted on the chip is removed as well. The regeneration of the chip for removing all specific or non-specific bound 
proteins was optimized to ensure the antibody functionality and allow a longevity of the chip without the need to recoat the chip prior to each analysis. We showed that the grafted antibody was able to resist for at least 100 analytical cycles including sample analysis and chip regeneration. This can be considered as a good level of longevity. Moreover, no sandwich detection was used in this case. The amount of antibody used was thus extremely low, limiting the costs associated to their use. From that, we estimated the cost of consumables for one analysis by the three methods studied here, in regards with their respective characteristics of throughput, handling complexity and time consumption (cf. Table 2). The estimated price per sample was calculated excluding the instruments prices, it comprised reagents, antibodies, buffers and supports (chip for SPR, gel support for $\mu C$-IE, ELISA plate). The price of a SPR analysis was found to be significantly lower than ELISA's, for an automated method allowing the detection of several fragments. The throughput of SPR is lower than other methods but presents the advantage to be easy-to-use and fully automated. The recently renovated $\mu \mathrm{C}$-IE [18] offering a high throughput use (35 samples per plate) allows a direct visualization of the native $\mathrm{C} 3$ and its fragments, providing a global vision of $\mathrm{C} 3$ status, in contrast with the ELISA method. Among other advantages, the $\mu \mathrm{C}$-IE method is much less expensive than the ELISA test. The inherent drawback of this method is its higher time consumption. However, it still can be applied for a deeper understanding of complement activation behavior and concentration studies $[18,37,38]$.

Table 2: Comparison of SPR, $\mu$ C-IE and ELISA methodological aspects towards a routine assessment of complement activation by nanomedicines. Advantages and drawbacks.

\begin{tabular}{|c|c|c|c|}
\hline & SPR & $\mu C$-IE & ELISA \\
\hline Cost/sample & $1.75 €$ & $0.14 €^{(20)}$ & $6 €$ \\
\hline Throughput & 7 samples/hour & 35 samples/ 6 hours & 96 samples/3 hours \\
\hline Handling & Easy & $\begin{array}{l}\text { Technical for gel } \\
\text { preparation (45 min) }\end{array}$ & $\begin{array}{l}\text { Not complex. } \\
\text { Pipetting can be } \\
\text { automated }\end{array}$ \\
\hline Time consumption & Fully automated & $\begin{array}{l}\text { Gel preparation - No } \\
\text { manipulation during } \\
\text { electrophoresis (5h) } \\
\text { - Gel staining }\end{array}$ & Full 3 hours \\
\hline $\begin{array}{l}\text { Information } \\
\text { obtained }\end{array}$ & $\begin{array}{c}\text { Multiplex fragment } \\
\text { quantitation (C3a, C3) }\end{array}$ & $\begin{array}{c}\text { Balance } \\
\text { C3/fragments }\end{array}$ & $\begin{array}{c}\text { Single fragment } \\
\text { quantitation (iC3b) }\end{array}$ \\
\hline
\end{tabular}

The SPR method was challenged to monitor complement activation triggered by a series of 5 nanoparticles for which we had previously established their complement activation status using the $\mu \mathrm{C}$-IE [37]. All nanoparticles were composed of a core of poly(isobutylcyanoacrylate) covered by a neutral polysaccharide, dextran, which conferred almost neutral charge on nanoparticle surface as acknowledged by the low value of the zeta potential. The analysis of complement activation of our five well known NPs showed consistent results between $\mu$ C-IE and SPR measurements. In contrast, the 
ELISA methods showed a very high background for all serum samples incubated with nanoparticles independently of the concentration and type of nanosystem. This unexplained result hampered the evaluation of complement activation produced by the different type of nanoparticles considered in this work. The ELISA test measured the protein iC3b which is a different fragment from those detected by the two other methods. Evaluating this fragment that results from the cleavage of the C3 protein into an inactivated $\mathrm{C} 3 \mathrm{~b}$ fragment could provide a very different insight of complement activation. However, the results obtained with the ELISA method could not be compared those obtained with SPR and $\mu \mathrm{C}-\mathrm{IE}$.

For a control quality analysis of complement activation, SPR could be the candidate of choice due to its simplicity and its possible automation. Nevertheless, while working with specific fragments, it should be verified that no bias or artefacts were interfering with the detection of the analyte. Indeed, in the present study we evaluated the influence of the preparation of a sample prior to its analysis by SPR. Indeed, in the literature, assessment of complement activation produced by nanoparticles is described on both serum samples still containing the nanoparticles [17] and on serum samples obtained after removal of the nanoparticles [39]. It is generally not explained why analysis is performed on either serum although it has been described that several C3 fragments has the tendency to adsorb on polymer surfaces [34]. Our study including the removal of NPs showed that the procedure when dealing with assessment of NP-protein interaction and protein detection is very important. C3a was found to adsorb strongly on R1, R2 and R3 NPs, all bearing a brush conformation of dextran. On the contrary A1 did not adsorb C3a while A2 was adsorbing in a less extent than nanoparticles of the R series. The adsorption of C3a onto certain polymer surfaces has already been mentioned [32-34] and was confirmed in our study. It is noteworthy that it seems hard to predict this specific adsorption from generally known characteristics of the nanoparticles (i.e. composition, size and zeta potential). Indeed, in our study, all nanoparticles were coated with dextran and only a slight modification on dextran chain architecture between A1 and A2 leads to different behaviors of C3a adsorption. This highlights the fact that careful and critical study of results must be done when dealing with detection of a specific marker for detection of complement but also in other cases when dealing with nanoparticles. As suggested by the results, the removal of NPs prior to analysis may generate bias leading to false negative results that may have dramatic consequence when evaluating the safety of a nanomaterial. When NPs were still in the medium, C3a could be recognized by antibodies grafted on the SPR chip, supposing that this fragment might be at the external surface of NPs in the soft corona and could be readily intercepted by antibodies grafted on the chip surface. The test in presence of CVF confirmed the fact that a specific adsorption of C3a occurred on NPs. Surprisingly, A1 did not adsorb C3a at all, while these NPs are known to adsorb protein of much larger size (data not published). Here, the C3a fragment mostly adsorbed on nanoparticles showing a lower surface curvature. In the context of characterization of interactions between nanomedicine and proteins, this is an insight into the specific adsorption of a small protein of the complement system. Among the known parameters affecting the adsorption, NPs' surface curvature and architecture are known to be of importance [40-42]. Here, these two parameters have a strong influence on C3a deposition onto nanoparticle surface. Results obtained from this part of the work brought new evidences that confirm the high complexity of phenomena governing protein adsorption onto NPs and the need for a better understanding of these interactions influencing the in vivo fate of nanoparticles. 


\section{Conclusion}

A SPR based method for the evaluation of complement activation by nanoparticles through C3 protein fragmentation has been developed in this work. The optimized conditions allowed for a prolonged use of the chip after a single antibody immobilization. Results provided by this method were consistent with those of the multi-crossed 2D immune-electrophoresis method, previously validated for complement activation evaluation. In contrast, no comparison with ELISA could be established due to a high non-specific background found with this method. The evidence of an artefact based on possible fragment adsorption onto NPs was shown by SPR considering analysis of samples containing or not the nanoparticles. This warn about caution required when performing complement activation analysis evaluating nanoparticles. Finally, the SPR method is a rapid and automatable which gave consistent results in terms of complement activation upon direct sample analysis without need of nanoparticle removal. This method could be the method of choice for a daily assessment of complement activation by nanomedicines and nanomaterials in general.

\section{Acknowledgements}

The authors acknowledge:

The financial support from BPI-France, Project NICE.

IPSIT Platform, Intermol (Chatenay-Malabry, France) for SPR analysis.

This work has benefited from the platform and expertise of the Electron Microscopy Facility if I2BC, CNRS, Gif sur Yvette, France.

\section{Bibliography}

1. Merle NS, Church SE, Fremeaux-Bacchi V, Roumenina LT. Complement System Part I-Molecular Mechanisms of Activation and Regulation. Front Immunol. 2015;6:262.

2. Merle NS, Noe R, Halbwachs-Mecarelli L, Fremeaux-Bacchi V, Roumenina LT. Complement System Part II: Role in Immunity. Front Immunol. 2015;6:257.

3. Dobrovolskaia MA, Aggarwal P, Hall JB, McNeil SE. Preclinical Studies To Understand Nanoparticle Interaction with the Immune System and Its Potential Effects on Nanoparticle Biodistribution. Mol. Pharm. 2008;5:487-95.

4. Nilsson B, Ekdahl KN, Mollnes TE, Lambris JD. The role of complement in biomaterial-induced inflammation. Mol. Immunol. 2007;44:82-94.

5. Szebeni J. Complement activation-related pseudoallergy: a new class of drug-induced acute immune toxicity. Toxicology. 2005;216:106-21.

6. Szebeni J. Hemocompatibility testing for nanomedicines and biologicals: predictive assays for complement mediated infusion reactions. Eur J Nanomed.. 2012;4:33.

7. Szeto GL, Lavik EB. Materials design at the interface of nanoparticles and innate immunity. J. Mater. Chem. B. 2016;4:1610-8.

8. Markiewski MM, DeAngelis RA, Benencia F, Ricklin-Lichtsteiner SK, Koutoulaki A, Gerard C, Coukos G, Lambris JD. Modulation of the anti-tumor immune response by complement. Nat. Immunol. 2008;9:1225-35.

9. Moghimi SM. Cancer nanomedicine and the complement system activation paradigm: Anaphylaxis and tumour growth. J Control Release. 2014;190:556-62. 
10.ISO 10993-4:2017: Biological evaluation of medical devices - Part 4: Selection of tests for interactions with blood. ISO; 2017.

11. ASTMF 1984: Standard practice for testing for whole complement activation in serum by solid materials; 2013.

12.FDA Guidance for Industry: Immunotoxicology Evaluation of Investigational New Drugs.; 2002. Available from https://www.fda.gov/downloads/drugs/guidancecomplianceregulatoryinformation/guidances/ucm 079239.pdf . Consulted on August 2017

13.EMA Reflection paper on the data requirements for intravenous liposomal products developed with reference to an innovator liposomal product.; 2013. Available from http://www.ema.europa.eu/docs/en GB/document library/Scientific guideline/2013/03/WC5001 40351.pdf. Consulted on August 2017

14. Harboe M, Thorgersen EB, Mollnes TE. Advances in assay of complement function and activation. Adv. Drug Deliver. Rev. 2011;63:976-87.

15.Salvador-Morales C, Sim RB. Complement activation. Handbook of immunological properties of engineered nanomaterials. 2013;357-84.

16. Mollnes TE, Jokiranta TS, Truedsson L, Nilsson B, Rodriguez de Cordoba S, Kirschfink M. Complement analysis in the 21st century. Mol. Immunol. 2007;44:3838-49.

17.Neun BW, Ilinskaya A, Dobrovolskaia MA, NCL Method ITA-5.2: Analysis of Complement Activation by EIA. 2015. https://ncl.cancer.gov/sites/default/files/protocols/NCL Method ITA-5.2.pdf Consulted on August 2017

18. Coty J-B, Varenne F, Vachon J-J, Vauthier C. Serial multiple crossed immunoelectrophoresis at a microscale: A stamp-sized 2D immunoanalysis of protein C3 activation caused by nanoparticles: Proteomics and 2-DE. Electrophoresis. 2016;37:2401-9.

19.Schneider CS, Bhargav AG, Perez JG, Wadajkar AS, Winkles JA, Woodworth GF, et al. Surface plasmon resonance as a high throughput method to evaluate specific and non-specific binding of nanotherapeutics. J Control Release. 2015;219:331-44.

20.Patra A, Ding T, Engudar G, Wang Y, Dykas MM, Liedberg B, et al. Component-Specific Analysis of Plasma Protein Corona Formation on Gold Nanoparticles Using Multiplexed Surface Plasmon Resonance. Small. 2016;12:1174-82.

21.Cedervall T, Lynch I, Lindman S, Berggård T, Thulin E, Nilsson H, et al. Understanding the nanoparticleprotein corona using methods to quantify exchange rates and affinities of proteins for nanoparticles. Proc. Natl. Acad. Sci. USA. 2007;104:2050-5.

22.Masson J-F, Battaglia TM, Cramer J, Beaudoin S, Sierks M, Booksh KS. Reduction of nonspecific protein binding on surface plasmon resonance biosensors. Anal Bioanal Chem. 2006;386:1951-9.

23.Seokheun C, Junseok C, Methods of reducing non-specific adsorption in microfluidic biosensors. J. Micromech. Microeng. 2010;20:075015.

24.Breault-Turcot J, Développement d'outils analytiques pour la détection de biomolécules directement dans des fluides sanguins. PhD thesis. 2015. Available from https://papyrus.bib.umontreal.ca/xmlui/bitstream/handle/1866/13566/Breault-

Turcot Julien 2015 these.pdf?sequence=6\&isAllowed=y Consulted on August 2017

25. Arima $\mathrm{Y}$, Toda $\mathrm{M}$, Iwata $\mathrm{H}$. Surface plasmon resonance in monitoring of complement activation on biomaterials. Adv. Drug Deliver. Rev. 2011;63:988-99.

26. Hirata I, Morimoto Y, Murakami Y, Iwata H, Kitano E, Kitamura H, et al. Study of complement activation on well-defined surfaces using surface plasmon resonance. Colloid Surface B. 2000;18:285-92.

27.Ricklin D, Lambris JD. Exploring the Complement Interaction Network Using Surface Plasmon Resonance. In: Lambris JD, editor. Current Topics in Innate Immunity. New York, NY: Springer New York; 2007. p. 260-78. 
28.Drake AW, Klakamp SL. A strategic and systematic approach for the determination of biosensor regeneration conditions. J Immunol Methods. 2011;371:165-9.

29.Andersson K, Areskoug D, Hardenborg E. Exploring buffer space for molecular interactions. J Mol Recognit. 1999;12:310-5.

30. Makaraviciute A, Ramanavicius A, Ramanaviciene A. Development of a reusable protein G based SPR immunosensor for direct human growth hormone detection in real samples. Anal. Methods. 2015;7:9875-84.

31.Szebeni J, Haima P, Hemocompatibility of medical devices, blood products, nanomedicines and biologicals. TECOmedical clinical and technical review. 2016.

32. Mares J, Thongboonkerd V, Tuma Z, Moravec J, Matejovic M. Specific adsorption of some complement activation proteins to polysulfone dialysis membranes during hemodialysis. Kidney Int. 2009;76:404-13.

33.Kandus A, Ponikvar R, Drinovec J, Kladnik S, Ivanovich P. Anaphylatoxins C3a and C5a adsorption on acrylonitrile membrane of hollow-fiber and plate dialyzer--an in vivo study. Int J Artif Organs. 1990;13:176-80.

34.Montdargent B, Toufik J, Carreno M-P, Labarre D, Jozefowicz M. Complement activation and adsorption of protein fragments by functionalized polymer surfaces in human serum. Biomaterials. 1992;13:571-6.

35.Bertholon I, Lesieur S, Labarre D, Besnard M, Vauthier C. Characterization of Dextran-Poly(isobutylcyanoacrylate) Copolymers Obtained by Redox Radical and Anionic Emulsion Polymerization. Macromolecules. 2006;39:3559-67.

36. Couvreur P, Kante B, Roland M, Guiot P, BAuduin P, Speiser P. Polycyanoacrylate nanocapsules as potential lysosomotropic carriers: preparation, morphological and sorptive properties. J Pharm Pharmacol. 1979;31:331-2.

37. Coty J-B, Eleamen Oliveira E, Vauthier C. Tuning complement activation and pathway through controlled molecular architecture of dextran chains in nanoparticle corona. Int J Pharm. 2017;532(2):769-778.

38.Nguyễn CH, Putaux J-L, Santoni G, Tfaili S, Fourmentin S, Coty J-B, et al. New nanoparticles obtained by co-assembly of amphiphilic cyclodextrins and nonlamellar single-chain lipids: Preparation and characterization. Int J Pharm. 2017;531(2):444-456.

39. Wibroe PP, Moghimi SM. Complement Sensing of Nanoparticles and Nanomedicines. In: Hepel M, Zhong C-J, editors. Functional Nanoparticles for Bioanalysis, Nanomedicine, and Bioelectronic Devices Volume 2. Washington, DC: American Chemical Society; 2012. p. 365-82.

40.Walkey CD, Olsen JB, Guo H, Emili A, Chan WCW. Nanoparticle Size and Surface Chemistry Determine Serum Protein Adsorption and Macrophage Uptake. J. Am. Chem. Soc. 2012;134:2139-47.

41.Gref R, Lück M, Quellec P, Marchand M, Dellacherie E, Harnisch S, et al. "Stealth" corona-core nanoparticles surface modified by polyethylene glycol (PEG): influences of the corona (PEG chain length and surface density) and of the core composition on phagocytic uptake and plasma protein adsorption. Colloid Surface B. 2000;18:301-13.

42.Pozzi D, Colapicchioni V, Caracciolo G, Piovesana S, Capriotti AL, Palchetti S, et al. Effect of polyethyleneglycol (PEG) chain length on the bio-nano-interactions between PEGylated lipid nanoparticles and biological fluids: from nanostructure to uptake in cancer cells. Nanoscale. 2014;6:2782. 


\title{
Supplementary information
}

\section{Assessment of complement activation by nanoparticles: development of a SPR based method and comparison with current high throughput methods}

\author{
J-B. Coty, M. Noiray, C. Vauthier* \\ Institut Galien Paris-Sud, CNRS, Univ. Paris-Sud, University Paris-Saclay, Châtenay-Malabry, \\ France. \\ *Correspondence: Dr. Christine Vauthier, CNRS UMR 8612, Institut Galien Paris Sud, Univ. Paris-Sud, \\ Université Paris-Saclay, 5, rue Jean-Baptiste Clément, 92296 Châtenay-Malabry, France. Fax: +33 \\ 146835946. E-mail: christine.vauthier@u-psud.fr
}




\section{S1: Size distribution of the nanoparticles used in the work}
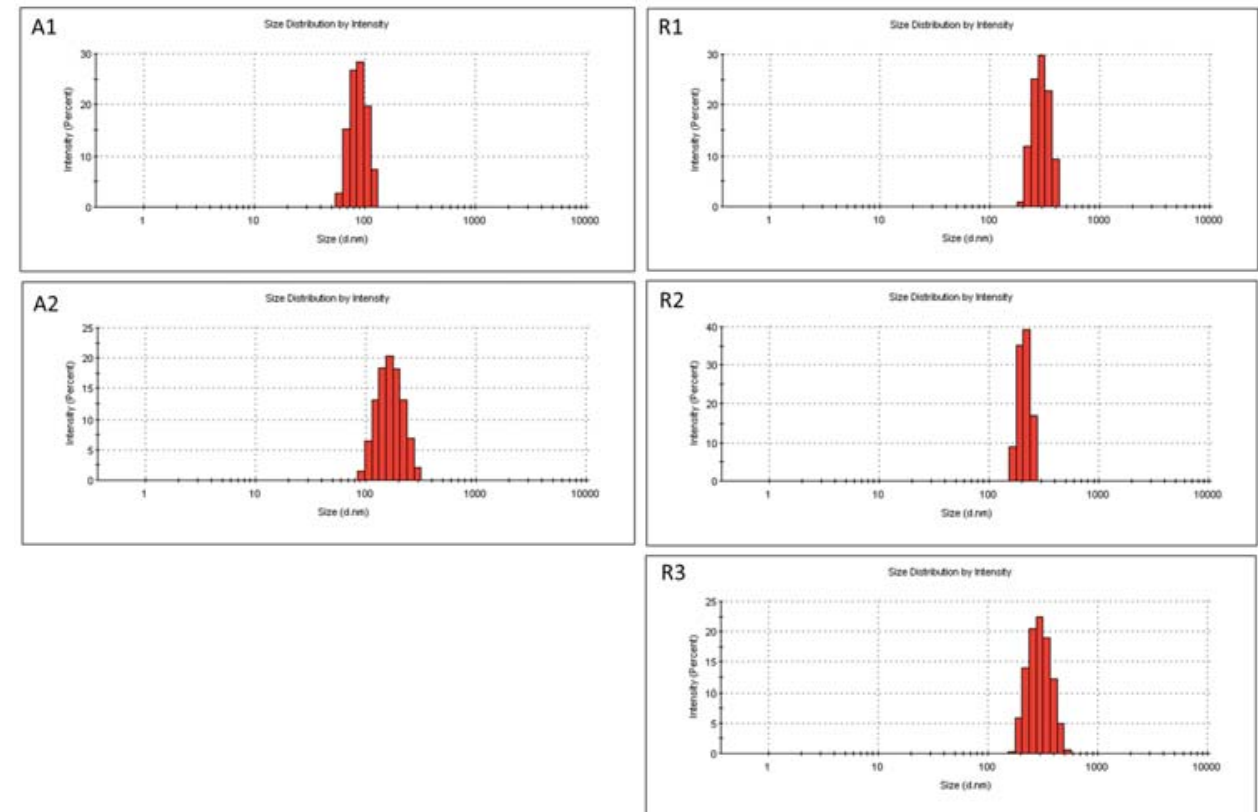

Figure S1: Size distribution in intensity of the different nanoparticle dispersions used in this work as evaluated by dynamic light scattering at $20^{\circ} \mathrm{C}$ (ZetaSizer Nano ZS90, Malvern Instruments, Orsay, France). Measurements were performed on dispersions diluted in MilliQ ${ }^{\circledR}$ water at $1 / 200$ (NPs R1 and R2), 1/100 (NPs A1), 1/50 (NPs R3) and 1/20 (NPs A2). 


\section{S2: Example of a sensorgram obtained by SPR}
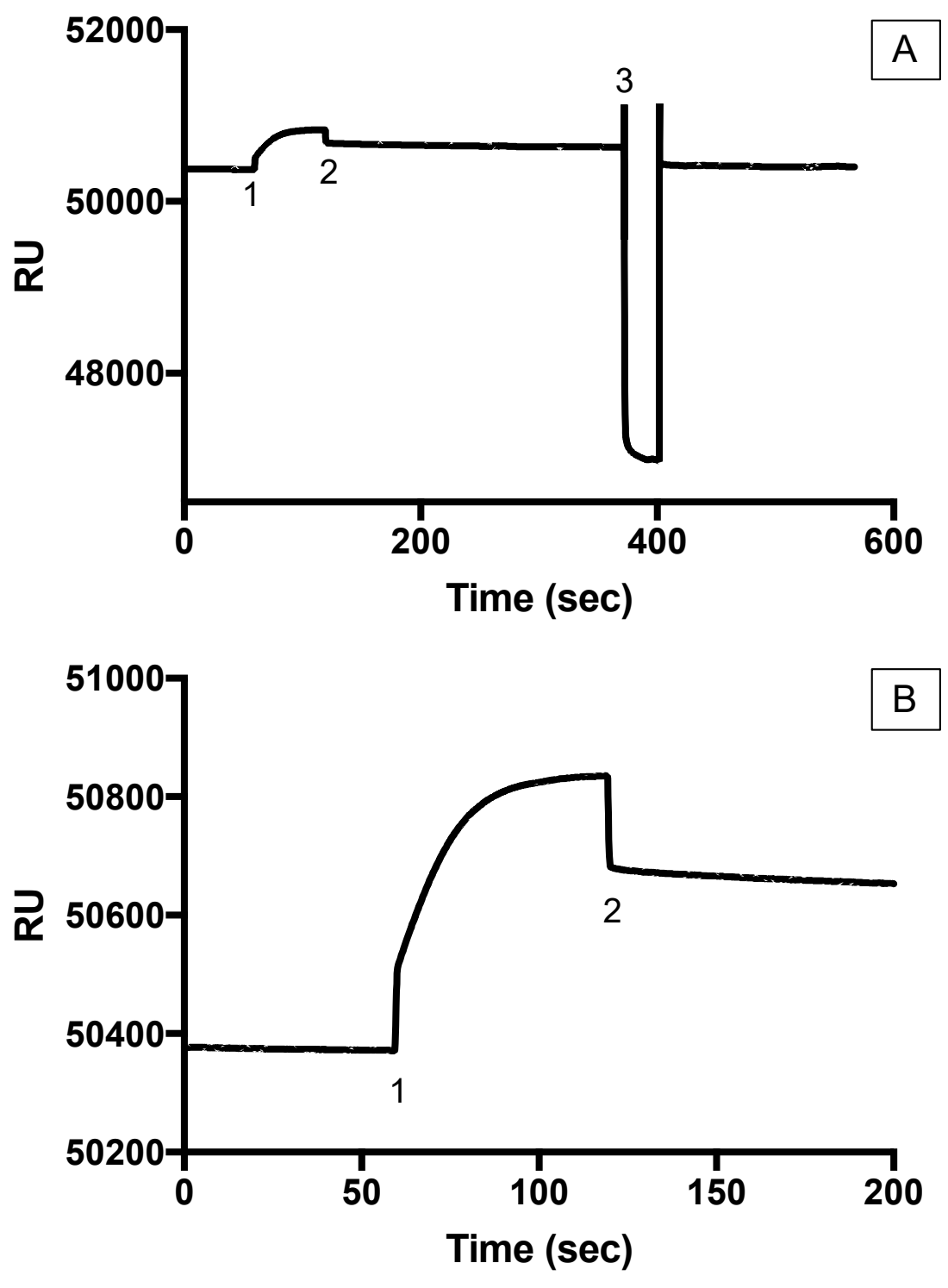

Figure S2: Sensorgram obtained on channel 2 (C3a) for the CVF sample.

(A) Full sensorgram. (B) Zoom of the binding/dissociation part.

1 represents the beginning of the association (injection of the sample)

2 represents the beginning of the dissociation (end of injection, buffer flow)

3 represents the beginning of the regeneration 


\section{S3: Effect of NPs removal on channels 1, 2 and 3}
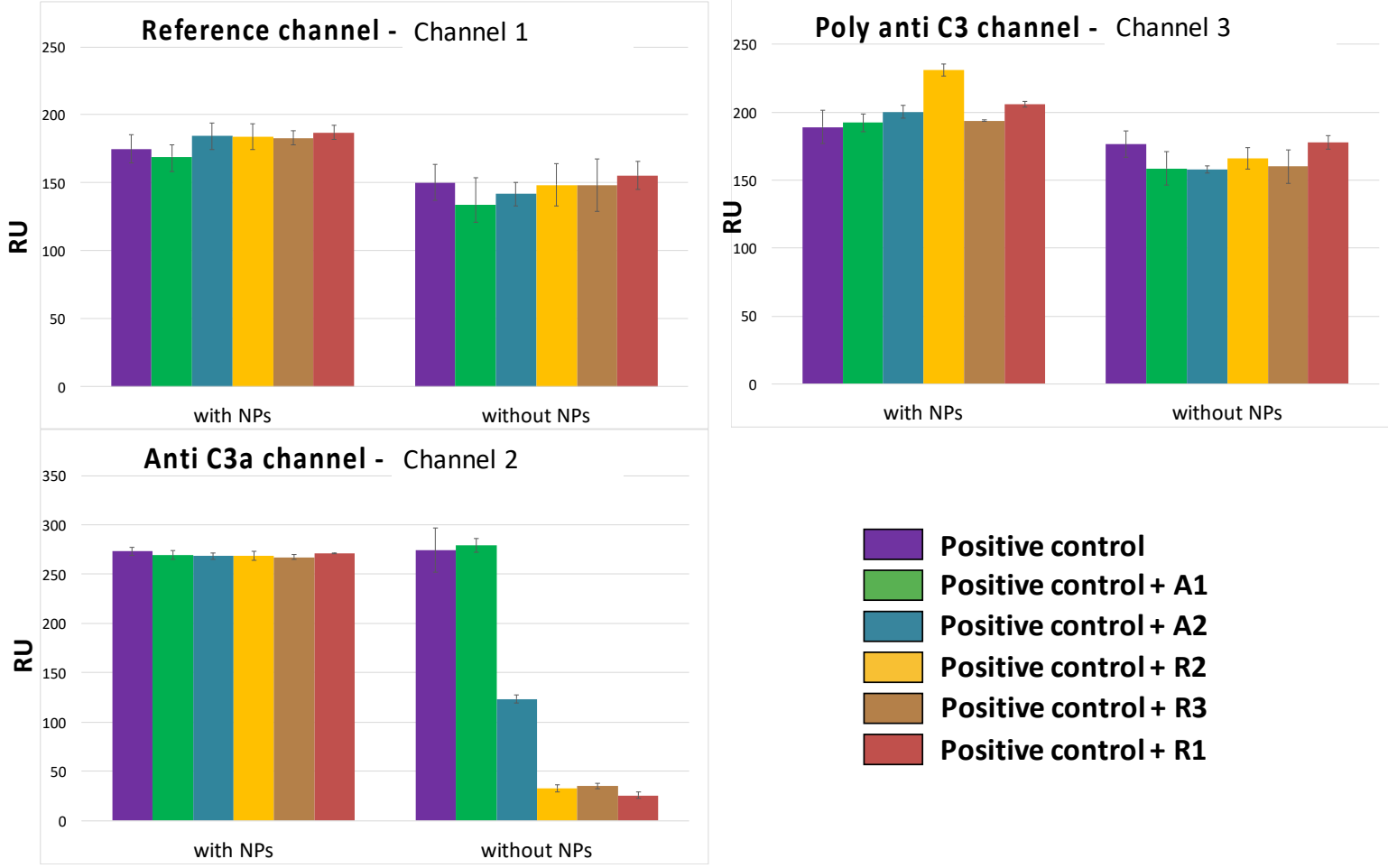

Figure S3: Responses obtained on channels 1, 2 and 3 for the study on NPS removal with positive controls. 